\title{
A DISCONTINUOUS $q$-FRACTIONAL BOUNDARY VALUE PROBLEM WITH EIGENPARAMETER DEPENDENT BOUNDARY CONDITIONS
}

\author{
F. AYCA CETINKAYA
}

Received 01 October, 2018

\begin{abstract}
This work aims to examine a boundary value problem which consists of a second order $q$-differential equation and eigenvalue dependent boundary conditions. We introduce a modified inner product in a suitable direct sum space and define a symmetric operator. We investigate the properties of eigenvalues and eigenfunctions and construct the Green's function.
\end{abstract}

2010 Mathematics Subject Classification: 34B09; 34L05

Keywords: $q$-calculus, boundary value problems, Green function, eigenvalues and eigenfunctions

\section{INTRODUCTION}

Recently, there has been a considerable attention on $q$-calculus and many papers subject to the boundary value problems consisting a Jackson $q$-derivative in the differential equation have appeared $([1-12,14,17,18])$. In $[6,8]$ the authors studied a $q$-analogue of the Sturm-Liouville eigenvalue problems and formulated a self-adjoint $q$-difference operator in a Hilbert space. Their results are applied and developed in different aspects. In [1,5], for instance, sampling theory associated with $q$-difference equations of the Sturm-Liouville type is considered. In [3,17] a regular $q$-fractional Sturm-Liouville problem which includes the left-sided Riemann-Liouville and rightsided Caputo $q$-fractional derivatives of the same order is formulated and the properties of eigenvalues and eigenfunctions are investigated. In [4] a Parseval equality and an expansion formula in eigenfunctions for a singular $q$-Sturm-Liouville operator on the whole line are established. In [2] the eigenvalues and the spectral singularities of non-selfadjoint $q$-difference equations of second order are investigated. In [11] a boundary value problem consisting a second order $q$-difference equation together with Dirichlet boundary conditions is reduced to an eigenvalue problem for a second order Euler $q$-difference equation by separation of variables and in [12] a $q$-SturmLiouville boundary value problem with a spectral parameter in the boundary condition is considered. For further studies related to the spectral analysis of $q$-differential 
equations and the Green's function, the readers are directed to [7,10,14,16,19-21] and the references therein.

Enlightened by these literature, in this paper, we study the boundary value problem

$$
\begin{gathered}
l(v):=-\frac{1}{q} D_{q^{-1}} D_{q} v(x)+r(x) v(x)=\eta w(x) v(x), \\
U_{1}(v):=\alpha_{1} v(0)+\alpha_{2} D_{q^{-1}} v(0)+\eta\left[\alpha_{3} v(0)+\alpha_{4} D_{q^{-1}} v(0)\right]=0, \\
U_{2}(v):=\beta_{1} v(\pi)+\beta_{2} D_{q^{-1}} v(\pi)+\eta\left[\beta_{3} v(\pi)+\beta_{4} D_{q^{-1}} v(\pi)\right]=0,
\end{gathered}
$$

where $q \in(0,1]$ is fixed, $r(\cdot)$ is a real valued function which is continuous at zero, $\eta$ is a complex parameter, $\alpha_{i} \neq 0, \beta_{j} \neq 0(i, j=1,2,3,4)$ are arbitrary real numbers and the function $w(x)$ is a positive piecewise continuous function such as

$$
w(x)= \begin{cases}w_{1}, & 0 \leq x<a, \\ w_{2}, & a<x \leq \pi\end{cases}
$$

The structure of the paper is as follows. In Section 2, we introduce notations, definitions and preliminary facts which are used throughout the paper. In Section 3 , we establish an operator-theoretic formulation for the boundary value problem (1.1)-(1.3) in the Hilbert space $L_{q, w}^{2}(0, \pi) \oplus \mathbb{C}^{2}$ and we give some of the virtues of eigenvalues and eigenfunctions. Section 4 is devoted to construct the Green's function for the boundary value problem (1.1)-(1.3) and to mention some of its properties. And, Section 5 gives the concluding remarks of the paper.

\section{PReliminaries}

In this section, we introduce some of the $q$-notations which will be used throughout the paper. We use the standard notations found in $[6,9,17]$.

The set of non-negative integers is denoted by $\mathbb{N}_{0}$, and the set of positive integers is denoted by $\mathbb{N}$. For $t>0$,

$$
A_{q, t}:=\left\{t q^{n}: n \in \mathbb{N}_{0}\right\}, \quad A_{q, t}^{*}:=A_{q, t} \cup\{0\},
$$

and

$$
\mathfrak{A}_{q, t}:=\left\{\mp t q^{n}: n \in \mathbb{N}_{0}\right\} .
$$

When $t=1$, we simply use $A_{q}, A_{q}^{*}$ and $\mathfrak{A}$ to denote $A_{q, 1}, A_{q, 1}^{*}$ and $\mathfrak{A}_{q, 1}$, respectively.

A set $S \subseteq \mathbb{R}$ is called a $q$-geometric set if, for every $x \in S, q x \in S$. Let $v$ be a real or complex valued function defined on a $q$-geometric set $S$. The $q$-difference operator is defined by

$$
D_{q} v(x):=\frac{v(x)-v(q x)}{x(1-q)}, \quad x \neq 0 .
$$

If $0 \in S$, the $q$-derivative of a function $v$ at zero is defined as

$$
D_{q} v(0):=\lim _{n \rightarrow \infty} \frac{v\left(x q^{n}\right)-v(0)}{x q^{n}}, \quad x \in S,
$$


if the limit exists and does not depend on $x$. Since the formulation of self-adjoint eigenvalue problems requires $D_{q}^{-1}$, we define it for $x \in S$ to be

$$
D_{q^{-1}} v(x)=\left\{\begin{array}{l}
\frac{v(x)-v\left(q^{-1} x\right)}{x\left(1-q^{-1}\right)}, \quad x \neq 0, \\
D_{q} v(0), \quad x=0
\end{array}\right.
$$

provided that $D_{q} v(0)$ exists. A right inverse, $q$-integration of the $q$-difference operator $D_{q}$ is defined by Jackson [15] as

$$
\int_{0}^{x} v(t) d_{q} t:=x(1-q) \sum_{n=0}^{\infty} q^{n} v\left(x q^{n}\right), x \in S,
$$

if the series converges. In general, the below equation is valid:

$$
\int_{a}^{b} v(t) d_{q}(t):=\int_{0}^{b} v(t) d_{q} t-\int_{0}^{a} v(t) d_{q} t, a, b \in S .
$$

There is no unique canonical choice for the $q$-integration over $[0, \infty)$. Hahn [13] defined the $q$-integration for a function $v$ over $[0, \infty)$ by

$$
\int_{0}^{\infty} v(t) d_{q} t=(1-q) \sum_{n=-\infty}^{\infty} q^{n} v\left(q^{n}\right)
$$

while Matsuo [18] defined $q$-integration on the interval $[0, \infty)$ with

$$
\int_{0}^{\infty} v(t) d_{q} t: b(1-q) \sum_{n=-\infty}^{\infty} q^{n} v\left(b q^{n}\right), b>0,
$$

provided that the series converges.

Consequently, the $q$-integration of a function $v$ defined on $\mathbb{R}$ can be defined as

$$
\int_{-\infty / b}^{\infty / b} v(t) d_{q} t=\frac{1-q}{b} \sum_{n=-\infty}^{\infty} q^{n}\left(v\left(q^{n} / b\right)+v\left(-q^{n} / b\right)\right), b>0
$$

provided that the series converges absolutely.

Definition 1. Let $v$ be a function defined on a $q$-geometric set $S$. We say that $v$ is $q$-integrable on $S$ if and only if $\int_{0}^{x} v(t) d_{q} t$ exists for all $x \in S$.

Let $S^{*}$ be a $q$-geometric set containing zero. A function $v$ defined on $S^{*}$ is called $q$-regular at zero if

$$
\lim _{n \rightarrow \infty} v\left(x q^{n}\right)=v(0)
$$

holds for all $v \in S^{*}$.

Let $C\left(S^{*}\right)$ denote the space of all $q$-regular at zero functions defined on $S^{*}$ with values in $\mathbb{R}$. $C\left(S^{*}\right)$, associated with the norm function

$$
\|v\|=\sup \left\{\left|v\left(x q^{n}\right)\right|: x \in S^{*}, n \in \mathbb{N}_{0}\right\},
$$


is a normed space. The $q$-integration by parts rule [8] is

$$
\int_{a}^{b} g(t) D_{q} f(t) d_{q} t=\left.(f g)\right|_{a} ^{b}+\int_{a}^{b} D_{q} g(t) f(q t) d_{q} t, \quad a, b \in S^{*}
$$

as $f, g \in C\left(S^{*}\right)$. While $p>0$, and $X$ is equivalent to $A_{q, t}$ or $A_{q, t}^{*}$, the space $L_{p}^{q}(X)$ is the normed space of all functions defined on $X$ such that

$$
\|v\|_{p}:=\left(\int_{0}^{x}|v|^{p} d_{q} t\right)^{1 / p}<\infty .
$$

If $p=2$, then $L_{q}^{2}(X)$ associated with the inner product

$$
\langle\varphi, \psi\rangle:=\int_{0}^{x} \varphi(t) \overline{\psi(t)} d_{q} t
$$

is a Hilbert space. By a weighted $L_{q, w}^{2}(X)$ space we mean the space of all functions $\varphi$ on $X$ such that

$$
\int_{0}^{x}|\varphi(t)|^{2} w(t) d_{q} t<\infty
$$

where $w$ is a positive function defined on $X$. The space $L_{q, w}^{2}(X)$ with the inner product

$$
\langle\varphi, \psi\rangle:=\int_{0}^{x} \varphi(t) \overline{\psi(t)} w(t) d_{q} t
$$

is a Hilbert space.

The space of all $q$-absolutely functions on $A_{q, t}^{*}$ is denoted by $A C_{q}\left(A_{q, t}^{*}\right)$ and defined as the space of all $q$-regular at zero functions $v$ satisfying

$$
\sum_{j=0}^{\infty}\left|v\left(t q^{j}\right)-v\left(t q^{j+1}\right)\right| \leq K
$$

for all $t \in A_{q, t}^{*}$, and $K$ is a constant depending on the function $v$ (c.f. [6], pg. 118, Definition 4.3.1), i. e. $A C_{q}\left(A_{q, t}^{*}\right) \subseteq C_{q}\left(A_{q, t}^{*}\right)$. The space $A C_{q}^{(n)}\left(A_{q, t}^{*}\right)(n \in \mathbb{N})$ is the space of all functions defined on $S^{*}$ such that $v, D_{q} v, \cdots, D_{q}^{n-1} v$ are $q$-regular at zero and $D_{q}^{n-1} v \in A C_{q}\left(A_{q, t}^{*}\right)$ (c.f. [6], pg. 119, Definition 4.3.2).

The following lemma which is needed in the sequel indicates that unlike the classical differential operator $\frac{d}{d x}, D_{q}$ is neither self adjoint nor skew self adjoint. Equation (2.4) below shows that the adjoint of $D_{q}$ is $-\frac{1}{q} D_{q^{-1}}$.

Lemma 1. Let $f(\cdot), g(\cdot)$ in $L_{q, w}^{2}(0, \pi)$ be defined on $\left[0, q^{-1} \pi\right]$. Then for $x \in$ $(0, \pi]$, we have

$$
\left(D_{q} g\right)\left(x q^{-1}\right)=D_{q, x q^{-1}} g\left(x q^{-1}\right)=D_{q^{-1}} g(x),
$$




$$
\begin{aligned}
\left\langle D_{q} f, g\right\rangle= & f(\pi) w(\pi) \overline{g\left(\pi q^{-1}\right)} \\
-\lim _{n \rightarrow \infty} f\left(\pi q^{n}\right) w\left(\left(\pi q^{n}\right) \overline{g\left(\pi q^{n-1}\right)}+\left\langle f,-\frac{1}{q} D_{q^{-1}} g\right\rangle\right. & \\
\left\langle-\frac{1}{q} D_{q^{-1}} f, g\right\rangle= & \lim _{n \rightarrow \infty} f\left(\pi q^{n-1}\right) w\left(\pi q^{n-1}\right) \overline{g\left(\pi q^{n}\right)} \\
& -f\left(\pi q^{-1}\right) w\left(\pi q^{-1}\right) \overline{g(\pi)}+\left\langle f, D_{q} g\right\rangle .
\end{aligned}
$$

Proof. The proof can be done similar to [8]. Indeed, relation (2.3) follows from

$$
D_{q^{-1}} g(x)=\frac{g(x)-g\left(q^{-1} x\right)}{x\left(1-q^{-1}\right)}=\frac{g\left(x q^{-1}\right)-g(x)}{x q^{-1}(1-q)}=\left(D_{q} g\left(x q^{-1}\right)\right) .
$$

Using the $q$-integration by parts formula (2.1), we obtain

$$
\begin{aligned}
\left\langle D_{q} f, g\right\rangle= & \int_{0}^{\pi} D_{q} f(x) w(x) \overline{g(x)}=f(\pi) w(\pi) \overline{g(\pi)} \\
& -\lim _{n \rightarrow \infty} f\left(\pi q^{n}\right) w\left(\pi q^{n}\right) \overline{g\left(\pi q^{n}\right)}-\int_{0}^{\pi} f(q t) w(q t) \overline{D_{q} g(t)} d_{q} t \\
= & f(\pi) w(\pi) \overline{g(\pi)}-\lim _{n \rightarrow \infty} f\left(\pi q^{n}\right) w\left(\pi q^{n}\right) \overline{g\left(\pi q^{n}\right)} \\
& -\int_{0}^{q \pi} f(t) w(t) \frac{1}{q} \overline{D_{q^{-1}} g(t)} d_{q} t \\
= & f(\pi) w(\pi) \overline{g(\pi)}-\lim _{n \rightarrow \infty} f\left(\pi q^{n}\right) w\left(\pi q^{n}\right) \overline{g\left(\pi q^{n}\right)} \\
& +\pi q^{-1}(1-q) f(\pi) w(\pi) \overline{D_{q^{-1}} g(\pi)}+\int_{0}^{\pi} f(t) w(t)-\frac{1}{q} D_{q^{-1}} g(t) d_{q} t \\
= & f(\pi) w(\pi) \overline{g\left(\pi q^{-1}\right)}-\lim _{n \rightarrow \infty} f\left(\pi q^{n}\right) w\left(\pi q^{n}\right) \overline{g\left(\pi q^{n}\right)}+\left\langle f,-\frac{1}{q} D_{q^{-1}} g\right\rangle
\end{aligned}
$$

proving (2.4). Equation (2.5) can be proven by using (2.4).

\section{OPERATOR THEORETIC FORMULATION}

In this section, we introduce a modified inner product in a suitable direct sum space, we define a symmetric operator on this space and we investigate some properties of eigenvalues and eigenfunctions.

In the Hilbert space $\mathscr{H}=L_{q, w}^{2}(0, \pi) \oplus \mathbb{C}^{2}$ let an inner product defined by

$$
(f, g):=\int_{0}^{\pi} f_{1}(x) g_{1}(x) w(x) d_{q} x+\frac{f_{2} \overline{g_{2}}}{\chi_{1}}+\frac{f_{3} \overline{g_{3}}}{\chi_{2}}
$$


where

$$
\begin{gathered}
f=\left(\begin{array}{c}
f_{1}(x) \\
f_{2} \\
f_{3}
\end{array}\right) \in \mathscr{H}, \quad g=\left(\begin{array}{c}
g_{1}(x) \\
g_{2} \\
g_{3}
\end{array}\right) \in \mathscr{H}, \quad \chi_{1}:=\alpha_{1} \alpha_{4}-\alpha_{2} \alpha_{3}>0, \\
\chi_{2}:=\beta_{1} \beta_{4}-\beta_{2} \beta_{3}>0 .
\end{gathered}
$$

We define the operator $A$

$$
A(f):=\left(\begin{array}{c}
-\frac{1}{q} D_{q^{-1}} D_{q} f_{1}(x)+r(x) f_{1}(x) \\
\alpha_{1} f_{1}(0)+\alpha_{2} D_{q^{-1}} f_{1}(0) \\
\beta_{1} f_{1}(\pi)+\beta_{2} D_{q^{-1}} f_{1}(\pi)
\end{array}\right)
$$

with the domain $D(A)$ which consists all the functions $f(x) \in \mathscr{H}$ that satisfy (1.2), (1.3) such that $f_{1}(x), D_{q^{-1}} f_{1}(x) \in A C_{q}\left[0, \pi q^{n}\right]$ and $l\left(f_{1}\right) \in L_{q, w}^{2}(0, \pi)$. Thus $A$ is the operator generated by the differential expression $l(f)=\eta w(x) f$ and the boundary conditions (1.2), (1.3).

Theorem 1. The operator $A$ is symmetric in the Hilbert space $\mathscr{H}$.

Proof. For each $f, g \in D(A)$ we have

$$
\begin{aligned}
(A f, g)-(f, A g)= & \int_{0}^{\pi} A f_{1}(x) \overline{g_{1}(x)} w(x) d_{q} x+\frac{A f_{2} \overline{g_{2}}}{\chi_{1}}+\frac{A f_{3} \overline{g_{3}}}{\chi_{2}} \\
& -\int_{0}^{\pi} f_{1}(x) \overline{A g_{1}(x)} w(x) d_{q} x-\frac{f_{2} \overline{A g_{2}}}{\chi_{1}}-\frac{f_{3} \overline{A g_{3}}}{\chi_{2}} \\
= & \int_{0}^{\pi}\left(-\frac{1}{q} D_{q^{-1}} D_{q} f_{1}(x)+r(x) f_{1}(x)\right) \overline{g_{1}(x)} w(x) d_{q}(x) \\
& -\int_{0}^{\pi} f_{1}(x) \overline{\left(-\frac{1}{q} D_{q^{-1}} D_{q} g_{1}(x)+r(x) g_{1}(x)\right)} w(x) d_{q}(x) \\
& +\frac{A f_{2} \overline{g_{2}}}{\chi_{1}}+\frac{A f_{3} \overline{g_{3}}}{\chi_{2}}-\frac{f_{2} \overline{A g_{2}}}{\chi_{1}}-\frac{f_{3} \overline{A g_{3}}}{\chi_{2}} .
\end{aligned}
$$

Applying equation (2.4) in Lemma 1 with $f(x)=D_{q} f_{1}(x), g(x)=g_{1}(x)$ to the first integral and keeping the realness of the function $r(x)$ in mind gives us

$$
\begin{aligned}
(A f, g)-(f, A g)= & \lim _{n \rightarrow \infty}\left(D_{q} f_{1}\right)\left(\pi q^{n-1}\right) w\left(\pi q^{n-1}\right) \overline{g_{1}\left(\pi q^{n}\right)} \\
& -\left(D_{q} f_{1}\right)\left(\pi q^{-1}\right) r\left(\pi q^{-1}\right) \overline{g_{1}(\pi)} \\
& +\left\langle D_{q} f_{1}, D_{q} g_{1}\right\rangle-\int_{0}^{\pi} f_{1}(x)\left(-\frac{1}{q} D_{q^{-1}} D_{q} g_{1}(x)\right) w(x) d_{q} x \\
& +\frac{A f_{2} \overline{g_{2}}}{\chi_{1}}+\frac{A f_{3} \overline{g_{3}}}{\chi_{2}}-\frac{f_{2} \overline{A g_{2}}}{\chi_{1}}-\frac{f_{3} \overline{A g_{3}}}{\chi_{2}}
\end{aligned}
$$


where $\langle\cdot, \cdot\rangle$ denotes the usual inner product in $L_{q}^{2}(0, \pi)$ which is defined in (2.2). Using (2.5) with $f(x)=f_{1}(x), g(x)=D_{q} g_{1}(x)$ to the term $\left\langle D_{q} f_{1}, D_{q} g_{1}\right\rangle$ in (3.1) we have

$$
\begin{aligned}
(A f, g)-(f, A g)= & {\left[f_{1}, g_{1}\right](\pi)-\lim _{n \rightarrow \infty}\left[f_{1}, g_{1}\right]\left(\pi q^{n}\right) } \\
& +\frac{A f_{2} \overline{g_{2}}}{\chi_{1}}+\frac{A f_{3} \overline{g_{3}}}{\chi_{2}}-\frac{f_{2} \overline{A g_{2}}}{\chi_{1}}-\frac{f_{3} \overline{A g_{3}}}{\chi_{2}}
\end{aligned}
$$

where

$$
[f, g](x):=f(x) w(x) \overline{D_{q^{-1}} g(x)}-D_{q^{-1}} f(x) w(x) \overline{g(x)} .
$$

If we use the definition for the domain of the operator $A$ we conclude that

$$
\frac{A f_{2} \overline{g_{2}}}{\chi_{1}}+\frac{A f_{3} \overline{g_{3}}}{\chi_{2}}-\frac{f_{2} \overline{A g_{2}}}{\chi_{1}}-\frac{f_{3} \overline{A g_{3}}}{\chi_{2}}=0 .
$$

Thus, we have

$$
(A f, g)-(f, A g)=\left[f_{1}, g_{1}\right](\pi)-\lim _{n \rightarrow \infty}\left[f_{1}, g_{1}\right]\left(\pi q^{n}\right) .
$$

Due to the fact that $f_{1}(x), g_{1}(x) \in C_{q}^{2}(0)$ satisfy the boundary conditions (1.2), (1.3) we have

$$
\left\{\begin{array}{l}
\alpha_{1} f_{1}(0)+\alpha_{2} D_{q^{-1}} f_{1}(0)+\eta\left[\alpha_{3} f_{1}(0)+\alpha_{4} D_{q^{-1}} f_{1}(0)\right]=0 \\
\alpha_{1} g_{1}(0)+\alpha_{2} D_{q^{-1}} g_{1}(0)+\eta\left[\alpha_{3} g_{1}(0)+\alpha_{4} D_{q^{-1}} g_{1}(0)\right]=0
\end{array}\right.
$$

The continuity of $f_{1}(x), g_{1}(x)$ at zero implies that

$$
\lim _{n \rightarrow \infty}\left[f_{1}, g_{1}\right]\left(\pi q^{n}\right)=\left[f_{1}, g_{1}\right](0)
$$

and we have

$$
(A f, g)-(f, A g)=\left[f_{1}, g_{1}\right](\pi)-\left[f_{1}, g_{1}\right](0) .
$$

Validity of the equations below follows from the fact that the functions $f(\cdot)$ and $g(\cdot)$ satisfy the boundary conditions (1.2), (1.3) and from formula (3.2):

$$
\begin{gathered}
{\left[f_{1}, g_{1}\right](0)=f_{1}(0) w(0) \overline{D_{q^{-1}} g_{1}(0)}-D_{q^{-1}} f_{1}(0) w(0) \overline{g_{1}(0)}=0,} \\
{\left[f_{1}, g_{1}\right](\pi)=f_{1}(\pi) w(\pi) \overline{D_{q^{-1}} g_{1}(\pi)}-D_{q^{-1}} f_{1}(\pi) w(\pi) \overline{g_{1}(\pi)}=0 .}
\end{gathered}
$$

Hence, the equation $(A f, g)-(f, A g)=0$ is satisfied and this completes the proof.

Definition 2. An $\eta$ which the boundary value problem (1.1)-(1.3) has a nontrivial solution is called an eigenvalue, and the corresponding solution, an eigenfunction. The multiplicity of an eigenvalue is defined to be the number of linearly independent solutions corresponding to it. In particular an eigenvalue is simple if and only if it has only one linearly independent solution. 
The eigenfunctions of the operator $A$ are in the form of

$$
\Phi\left(x, \eta_{n}\right)=\Phi_{n}:=\left(\begin{array}{c}
\varphi\left(x, \eta_{n}\right) \\
\alpha_{3} \varphi\left(0, \eta_{n}\right)+\alpha_{4} D_{q^{-1}} \varphi\left(0, \eta_{n}\right) \\
\beta_{3} \varphi\left(\pi, \eta_{n}\right)+\beta_{4} D_{q^{-1}} \varphi\left(\pi, \eta_{n}\right)
\end{array}\right) .
$$

Having the self-adjointness of the operator $A$ proven we have the following corollaries.

Corollary 1. The eigenfunctions $\Phi_{1}$ and $\Phi_{2}$ corresponding to the different eigenvalues are orthogonal.

Corollary 2. The eigenvalues of the boundary value problem (1.1)-(1.3) are real.

Now, let us denote

$$
\Delta(\eta):=\left|\begin{array}{ll}
U_{1}\left(\Phi_{1}\right) & U_{1}\left(\Phi_{2}\right) \\
U_{2}\left(\Phi_{1}\right) & U_{2}\left(\Phi_{2}\right)
\end{array}\right|
$$

where $\Phi_{i}(\cdot, \eta)$ are determined by the initial conditions

$$
D_{q}^{j-1} \Phi_{i}(\cdot, \eta)=\delta_{i j}, \quad(i=1,2, \eta \in \mathbb{C})
$$

as $\delta_{i j}$ refers to the Kronecker delta. The function $\Delta(\eta)$ is the characteristic function of the boundary value problem (1.1)-(1.3). It is an entire function with respect to $\eta$ and thus the eigenvalues of the boundary value problem (1.1)-(1.3) has an at most countable set of $\left\{\eta_{n}\right\}$ with no finite limit points.

In the following theorem, we prove that the eigenvalues of the boundary value problem (1.1)-(1.3) are the simple zeros of the characteristic function $\Delta(\eta)$.

Theorem 2. The eigenvalues of the boundary value problem (1.1)-(1.3) coincide with the simple zeros of $\Delta(\eta)$.

Proof. The proof follows similar steps as shown in Theorem 3.6 in [6] (pg. 83). Indeed, let us define the functions $\theta_{1}(\cdot, \eta)$ and $\theta_{2}(\cdot, \eta)$ as

$$
\left\{\begin{array}{l}
\theta_{1}(x, \eta):=U_{1}\left(\phi_{1}\right) \phi_{1}(x, \eta)-U_{1}\left(\phi_{1}\right) \phi_{2}(x, \eta), \\
\theta_{2}(x, \eta):=U_{2}\left(\phi_{2}\right) \phi_{1}(x, \eta)-U_{2}\left(\phi_{1}\right) \phi_{2}(x, \eta) .
\end{array}\right.
$$

Hence it can easily be seen that the functions $\theta_{1}(\cdot, \eta)$ and $\theta_{2}(\cdot, \eta)$ are the two solutions of equation (1.1) which satisfy the initial conditions

$$
\begin{cases}\theta_{1}(0, \eta)=\alpha_{2}+\eta \alpha_{4}, & D_{q^{-1}} \theta_{1}(0, \eta)=-\left(\alpha_{1}+\eta \alpha_{3}\right), \\ \theta_{2}(\pi, \eta)=\beta_{2}+\eta \beta_{4}, & D_{q^{-1}} \theta_{2}(\pi, \eta)=-\left(\beta_{1}+\eta \beta_{3}\right) .\end{cases}
$$

Now, let $\eta_{0}$ be an eigenvalue of the boundary value problem (1.1)-(1.3). The equation below

$$
W_{q}\left(\theta_{1}(\cdot, \eta), \theta_{2}(\cdot, \eta)\right)=\Delta(\eta) W_{q}\left(\phi_{1}(\cdot, \eta), \phi_{2}(\cdot, \eta)\right)(x)=\Delta(\eta)
$$

leads us to the fact that the real valued functions $\theta_{i}\left(x, \eta_{0}\right)(i=1,2)$ are linearly dependent:

$$
\theta_{1}\left(x, \eta_{0}\right)=k_{0} \theta_{2}\left(x, \eta_{0}\right),\left(k_{0} \neq 0\right) .
$$


Using (3.4) and (3.5) implies

$$
\left\{\begin{array}{l}
\theta_{1}\left(\pi, \eta_{0}\right)=k_{0} \theta_{2}\left(\pi, \eta_{0}\right)=k_{0}\left(\beta_{2}+\eta \beta_{4}\right), \\
D_{q^{-1}} \theta_{1}(\pi, \eta)=k_{0} D_{q^{-1}} \theta_{2}\left(\pi, \eta_{0}\right)=-k_{0}\left(\beta_{1}+\eta \beta_{3}\right) .
\end{array}\right.
$$

By applying $q$-Lagrange identity to the functions $\theta_{1}(x, \eta)$ and $\theta_{1}\left(x, \eta_{0}\right)$ we obtain

$$
\begin{aligned}
\left(\eta-\eta_{0}\right) & \int_{0}^{\pi} \theta_{1}(x, \eta) \theta_{1}\left(x, \eta_{0}\right) d_{q} x \\
& =\theta_{1}(\pi, \eta) D_{q^{-1}} \theta_{1}\left(\pi, \eta_{0}\right)-D_{q^{-1}} \theta_{1}(\pi, \eta) \theta_{1}(\pi, \eta) \\
& =k_{0}\left(\theta_{1}(\pi, \eta) D_{q^{-1}} \theta_{2}\left(\pi, \eta_{0}\right)-\theta_{2}\left(\pi, \eta_{0}\right) D_{q^{-1}} \theta_{1}(\pi, \eta)\right) \\
& =k_{0} W_{q}\left(\theta_{1}(\cdot, \eta), \theta_{2}\left(\cdot, \eta_{0}\right)\right)\left(q^{-1} \pi\right) \\
& =k_{0} \Delta(\eta)
\end{aligned}
$$

Since $\Delta(\eta)$ is an entire function of $\eta$ we have the opportunity to write the expression below:

$$
\frac{d}{d \eta} \Delta(\eta)=\lim _{\eta \rightarrow \eta_{0}} \frac{\Delta(\eta)-\Delta_{0}(\eta)}{\eta-\eta_{0}}=\frac{1}{k_{0}} \int_{0}^{\pi} \theta_{1}^{2}\left(x, \eta_{0}\right) d_{q} x \neq 0 .
$$

The simplicity of the zeros of the function $\Delta(\eta)$ is the direct result of (3.6).

\section{Construction of the $q$-TYPE GREen's FUnCtion}

The $q$-type Green's function arises when we pursue a solution of the inhomogeneous boundary value problem

$$
\begin{gathered}
l(v):=-\frac{1}{q} D_{q^{-1}} D_{q} v(x)+\{-\eta w(x)+r(x)\} v(x)=f(x), \quad x \in[0, \pi] \\
U_{1}(v):=\alpha_{1} v(0)+\alpha_{2} D_{q^{-1}} y(0)+\eta\left[\alpha_{3} v(0)+\alpha_{4} D_{q^{-1}} v(0)\right]=f_{2}, \\
U_{2}(v):=\beta_{1} v(\pi)+\beta_{2} D_{q^{-1}} v(\pi)+\eta\left[\beta_{3} v(\pi)+\beta_{4} D_{q^{-1}} v(\pi)\right]=f_{3},
\end{gathered}
$$

where $f(x) \in L_{q, w}^{2}(0, \pi)$.

Theorem 3. Assume that $\eta$ is not an eigenvalue of the boundary value problem (1.1)-(1.3). Let $\phi(\cdot, \eta)$ satisfy the $q$-difference equation (4.1) and the boundary conditions (4.2)-(4.3) where $f(x) \in L_{q, w}^{2}(0, \pi)$. Then

$$
\begin{aligned}
\phi(x, \eta)= & \int_{0}^{\pi} G(x, t, \eta) f(t) d_{q} t+\frac{f_{2}\left(\alpha_{3} G(0, \cdot, \eta)+\alpha_{4} D_{q^{-1}} G(0, \cdot, \eta)\right)}{\chi_{1}} \\
& +\frac{f_{3}\left(\beta_{3} G(\pi, \cdot, \eta)+\beta_{4} D_{q^{-1}} G(\pi, \cdot, \eta)\right)}{\chi_{2}}
\end{aligned}
$$

where $G(x, t ; \eta)$ is the Green's function of the inhomogeneous boundary value problem (4.1)-(4.3) defined by

$$
G(x, t ; \eta)=-\frac{1}{\Delta(\eta)}\left\{\begin{array}{l}
\theta_{2}(x, \eta) \theta_{1}(t, \eta), t \leq x \\
\theta_{1}(x, \eta) \theta_{2}(t, \eta), x \leq t .
\end{array}\right.
$$


Conversely, the function $\phi(x, \eta)$ defined by (4.4) satisfies (4.1) and (4.2), (4.3).

Proof. We shall search the solution of the inhomogeneous boundary value problem (4.1)-(4.3) as

$$
\phi(x, \eta)=c_{1}(x) \theta_{1}(x, \eta)+c_{2}(x) \theta_{2}(x, \eta)
$$

where the functions $c_{1}(x)$ and $c_{2}(x)$ are the solutions of the system of equations

$$
\left\{\begin{array}{l}
D_{q, x} c_{1}(x) \theta_{1}(x, \eta)+D_{q, x} c_{2}(x) \theta_{2}(x, \eta)=0 \\
D_{q, x} c_{1}(x) D_{q, x} \theta_{1}(x, \eta)+D_{q, x} c_{2}(x) D_{q, x} \theta_{2}(x, \eta)=f(x)
\end{array}\right.
$$

If the functions $D_{q, x} c_{i}(x)(i=1,2)$ are $q$-integrable on $[0, t]$ then

$$
\lim _{n \rightarrow \infty} t q^{n} \theta_{i}\left(t q^{n+1}, \eta\right) f\left(t q^{n+1}\right)=0,(i=1,2) .
$$

Now, let us define the $q$-geometric set $S_{f}$ by

$$
S_{f}:=\left\{x \in[0, \pi]: \lim _{n \rightarrow \infty} x q^{n}\left|f\left(x q^{n}\right)\right|^{2}=0\right\} .
$$

Since, $f \in L_{q, w}^{2}(0, \pi)$ the set $S_{f}$ is a $q$-geometric set containing $\left\{\pi q^{m}: m \in \mathbb{N}_{0}\right\}$. Hence, $D_{q} c_{i}(\cdot),(i=1,2)$ are $q$-integrable on $[0, x]$ for all $x \in S_{f}$ and the solutions of (4.6) are

$$
\left\{\begin{array}{l}
c_{1}(x)=\tilde{c_{1}}+\frac{q}{\Delta(\eta)} \int_{0}^{x} \theta_{2}(q t, \eta) f(q t) w(q t) d_{q} t, \\
c_{2}(x)=\tilde{c_{2}}+\frac{q}{\Delta(\eta)} \int_{x}^{\pi} \theta_{1}(q t, \eta) f(q t) w(q t) d_{q} t,
\end{array}\right.
$$

where $\tilde{c_{1}}, \tilde{c_{2}}$ are unknown constants and $x \in S_{f}$. Substituting (4.7) into (4.5) and taking (4.2), (4.3) into consideration leads us to (4.4). Conversely, if $\phi(x, \eta)$ is given by (4.4), then it is a solution of (4.1) which satisfies the boundary conditions (4.2), (4.3) and this completes the proof.

The following theorem lists a number of properties of the Green's function.

Theorem 4. Green's function has the following properties:

(1) $G(x, t, \eta)$ is continuous at the point $(0,0)$.

(2) $G(x, t, \eta)=G(t, x, \eta)$.

(3) For each fixed $t \in(0, q \pi], G(x, t, \eta)$ satisfies the $q$-difference equation (4.1) in the intervals $[0, t),(t, \pi]$ and it also satisfies the boundary conditions (4.2)(4.3).

Proof. The proof can easily be obtained by using a similar procedure to [6].

\section{CONCLUSION}

In this work we investigate a boundary value problem which consists of a second order $q$-differential equation and eigenvalue dependent boundary conditions. We introduce a modified inner product in a suitable direct sum space $L_{q, w}^{2}(0, \pi) \oplus \mathbb{C}^{2}$. We define the operator $A$ on this space and prove that it is symmetric. We provide some 
of the properties of eigenvalues and eigenfunctions and we construct the Green's function.

\section{ACKNOWLEDGEMENTS}

We would like to thank the reviewers for their valuable suggestions and comments, which improved the completeness of the paper.

\section{REFERENCES}

[1] L. Abreu, "Sampling theory associated with $q$-difference equations of the Sturm-Liouville type," J. Phys. A, vol. 38, no. 48, pp. 10311-10319, 2005, doi: 10.1088/0305-4470/38/48/005.

[2] M. Adivar and M. Bohner, "Spectral analysis of a $q$-difference equations with spectral singularities," Math. Comput. Model., vol. 43, no. 7-8, pp. 695-703, 2006, doi: 10.1016/j.mcm.2005.04.014.

[3] M. A. Al-Towaib, “A $q$-fractional approach to the regular Sturm-Liouville problems," Electron. J. Differential Equations, vol. 2017, no. 88, pp. 1-13, 2017.

[4] B. P. Allahverdiev and H. Tuna, "An expansion theorem for $q$-Sturm-Liouville operators on the whole line," Turkish J. Math., vol. 42, no. 3, pp. 1060-1071, 2018, doi: 10.3906/mat-1705-22.

[5] M. H. Annaby, J. Bustoz, and M. Ismail, "On sampling theory and basic Sturm-Liouville systems," J. Comput. Appl. Math., vol. 206, pp. 73-85, 2007, doi: 10.1016/j.cam.2006.05.024.

[6] M. H. Annaby and Z. S. Mansour, "Basic Sturm-Liouville problems," J. Phys. A: Math. Gen., vol. 38, pp. 3775-3797, 2005, doi: 10.1088/0305-4470/38/17/005.

[7] M. H. Annaby and Z. S. Mansour, "Asymptotic formulae for eigenvalues and eigenfunction of $q$-Sturm-Liouville problems," Math. Nachr., vol. 284, no. 4, pp. 443-470, 2011, doi: 10.1002/mana.200810037.

[8] M. H. Annaby and Z. S. Mansour, q-fractional calculus and equations. New York: Springer, 2012.

[9] M. H. Annaby, Z. S. Mansour, and I. A. Soliman, " $q$-Titchmarsh-Weyl theory:Series expansion," Nagoya Math. J., vol. 205, pp. 67-118, 2012, doi: 10.1215/00277630-1543787.

[10] M. B. Bekker, M. J. Bohner, A. N. Herega, and H. Voulov, "Spectral analysis of a $q$-difference equation and its applications," Appl. Math. Lett., vol. 22, no. 4, pp. 521-527, 2009, doi: 10.1088/1751-8113/43/14/145207.

[11] M. Bohner and T. Hudson, "Euler-type boundary value problems in quantum calculus," IJAMAS, vol. 9, no. J07, pp. 19-23, 2007.

[12] A. Eryilmaz, "Spectral analysis of a $q$-Sturm-Liouville problem with the spectral parameter in the boundary condition,” J. Funct. Spaces, vol. 2012, p. 17 pages, 2012, doi: 10.1155/2012/736437.

[13] W. Hahn, "Beitrage zur theorie der heineschen reihen," Math. Nachr., vol. 2, pp. 340-379, 1949, doi: 10.1002/mana.19490020604.

[14] A. Huseynov and E. Bairamov, "An eigenvalue problem for quadratic pencils of $q$-difference equation and its applications," Appl. Math. Lett., vol. 22, no. 4, pp. 521-527, 2009, doi: 10.1016/j.aml.2008.07.003.

[15] F. H. Jackson, “On q-definite integrals," Quart. J. Pure Appl. Math., vol. 41, pp. 193-203, 1910, doi: $10.1017 /$ S00805800002751.

[16] F. F. Liao, "Periodic solutions of second order differential equations with vanishing Green's functions," Electron. J. Qual. Theory Differ. Equ., vol. 55, pp. 1-8, 2017, doi: 10.14232/ejqtde.2017.1.55.

[17] Z. S. Mansour, "On fractional q-Sturm-Liouville problems," J. Fixed Point Theory Appl., vol. 19, pp. 1591-1612, 2017, doi: 10.1007/s11784-016-0331-y. 
[18] A. Matsuo, "Jackson integrals of Jordan-Pochhammer type and quantum KnizhnikZamolodchikov equations," Comm. Math. Phys., vol. 151, pp. 263-273, 1993.

[19] S. Polidoro and M. A. Ragusa, "Hölder regularity for solutions of ultraparabolic equations in divergence form," Potential Analysis, vol. 14, no. 4, pp. 341-350, 2001, doi: 10.1023/A:1011261019736.

[20] S. Polidoro and M. A. Ragusa, "Harnack inequality for hypoelliptic ultraparabolic equations with singular lower order term," Rev. Mat. Iberoamericana, vol. 24, no. 3, pp. 1011-1046, 2008.

[21] A. Samoilenko, A. Boichuk, and S. Chuiko, "Hybrid difference-differential boundary value problems," Miskolc Mathematical Notes, vol. 18, no. 2, pp. 1015-1031, 2017, doi: 10/18514/MMN.2017.2280.

\section{Author's address}

\section{F. Ayca Cetinkaya}

Mersin University, Department of Mathematics, 33343 Mersin, Turkiye

E-mail address: faycacetinkaya@mersin.edu.tr 